\title{
Amyloid, thrombosis, and acute myocardial infarction in association with a bicuspid aortic valve
}

\author{
P H Groves, A G Douglas-Jones, R J C Hall
}

\begin{abstract}
A 34 year old man presented with an inferior non-Q-wave myocardial infarction. Echocardiography showed a bicuspid aortic valve with aortic outflow obstruction. Left coronary cusp morphology was normal but the right coronary cusp was grossly distorted and replaced by a mobile echodense mass encroaching upon the aortic valve orifice. The aortic valve was replaced and pathological analysis of the excised valve showed primary amyloid infiltration of the right coronary cusp but a normal left coronary cusp. The mass adherent to the right coronary leaflet had the histological appearances of organised thrombus and this was assumed to be the source of coronary embolism. This is the first reported case of primary valvar amyloid presenting with clinical sequelae and it illustrates the need for careful clinical assessment in young patients presenting with acute ischaemic syndromes.
\end{abstract}

(Br Heart f 1993;70:560-562)

Microscopic amyloid deposits associated with calcification are commonly found in chronically diseased heart valves. ${ }^{12}$ Isolated amyloid infiltration in the absence of valve scarring is less common and has not been reported in association with clinical sequelae. ${ }^{3} \mathrm{We}$ describe a unique case of a young fit man presenting with acute myocardial infarction secondary to embolism arising from a bicuspid aortic valve. The excised valve showed considerable amounts of primary amyloid deposit and overlying thrombus on one aortic leaflet only.

Department of Cardiology, University Hospital of Wales, Cardiff P H Groves R J C Hall

Department of Pathology, University Hospital of Wales, Cardiff

A G Douglas-Jones

Correspondence to:

Dr P H Groves, Department of Cardiology, University Hospital of Wales, Heat Park, Cardiff CF4 4XN. was no other previous medical history: he had not had rheumatic fever or a recent febrile illness. There were signs of mixed aortic valve disease and no clinical stigmata of infective endocarditis. An electrocardiogram showed deep inferior $T$ wave inversion and serial cardiac enzymes were diagnostic of myocardial infarction (peak creatine kinase $738 \mathrm{U} / 1-$ (normal $<195 \mathrm{U} / 1)$ ). The chest $x$-ray was normal and showed no valvar calcification. A cross sectional echocardiogram showed a bicuspid aortic valve with a normal left coronary cusp and gross distortion of the right coronary cusp, which was replaced by an echodense polypoid mass that was mobile and encroached upon the aortic valve orifice. The other cardiac valves were normal and no other intracardiac masses or thrombus were detected. The left ventricle was concentrically hypertrophied but not dilated and systolic contraction was good. Continuous wave Doppler echocardiography confirmed aortic stenosis and aortic regurgitation: the peak systolic gradient across the aortic valve was estimated from the Doppler signal to be 80 $\mathrm{mm} \mathrm{Hg}$ (modified Bernoulli equation).

The clinical diagnosis was inferior myocardial infarction, which was presumed to have resulted from a right coronary embolus arising from the abnormal aortic right coronary cusp. The patient was anticoagulated with warfarin, remained afebrile, and made an uncomplicated recovery. The electrocardiogram showed normalisation of the inferior $T$ waves without the development of $Q$ waves. Blood cultures were negative.

Six weeks later the aortic valve was replaced by a tilting disc prosthesis. Examination of the native aortic valve confirmed that it was originally bicuspid with congenital fusion of the right and non-coronary cusps. The right coronary portion of the fused cusp was totally replaced by a protuberant mass that impinged on the left coronary cusp and spread inferiorly towards the adjacent left ventricular wall. There was no macroscopic evidence of infection. The coronary orifices were normally situated and the cooling pattern of the myocardium during the administration of cold cardioplegia was uniform, implying the absence of obstructive coronary artery disease. Specimens from the excised valve were sterile on culture.

Postoperative recovery was uneventful and the patient has since remained fit and well. An exercise stress test performed two months after aortic valve replacement was normal. 
Excised aortic valve after formalin fixation showing the large polypoid mass arising from the superior surface of the right coronary cusp. $B a r=1 \mathrm{~cm}$.

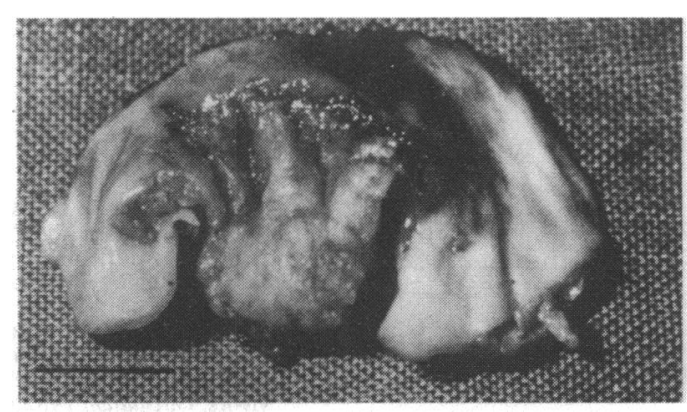

\section{Pathological examination}

Macroscopically, the left coronary cusp of the excised aortic valve appeared normal, but a large polypoid irregular mass with a wide base $(2.3 \times 2.0 \mathrm{~cm})$ and rose $1.2 \mathrm{~cm}$ above the valve surface of the right coronary cusp (figure). The cut surface of this mass had an homogenous pale translucent appearance. The valve was fixed in $10 \%$ buffered formalin and sections were taken for histological analysis.

Staining with haematoxylin and eosin and with elastic van Gieson stain showed that the normal architecture of the right coronary cusp was disrupted by eosinophilic acellular material. There was no evidence of inflammation or calcification within the valve leaflet. Stains for bacteria (gram), fungi (periodic $\mathrm{acid} / \mathrm{Schiff}$ ) and mycobacterium (ZiehlNeelsen) were negative. The eosinophilic deposits stained with Congo red, showed green birefringence under cross-polarised light, stained with sodium sulphate alcian Blue (SAB), and showed fluorescence on thioflavin $T$ staining, indicating that the intravalvar material was amyloid. Immunocytochemistry showed positive staining with antibodies against substance $P$ but no reactivity for anti-amyloid AA, confirming that these were primary amyloid deposits. ${ }^{4}$ There was no evidence of amyloid within the acellular polypoid mass that was attached to the right coronary cusp. This had the staining characteristics of post-mature fibrin ${ }^{5}$ and samples subjected to transmission electron microscopy showed degenerate fibrillar material and cellular debris. Histological examination of the left coronary cusp showed mild thickening but normal valve architecture with no amyloid material detected either by light or electron microscopy.

\section{Discussion}

We believe that this is the first reported case of primary amyloid involving a bicuspid aortic valve in which thrombosis and presumed embolism led to myocardial infarction. This case has several interesting and unusual features. The acute illness was preceded by a three month history of chest discomfort on exertion, implying the presence of haemodynamically significant aortic valve obstruction. The symptoms resolved after aortic valve replacement. The pathological evidence suggested that aortic valve obstruction was caused by the prolapse of the protuberant mass on the right coronary cusp into the left ventricular outflow tract rather than by congenital bicuspid aortic valve stenosis. The histological appearance of the mass indicated that it was made up of organised thrombus. There was no clinical, microbiological, or histological evidence of infective endocarditis. Why thrombus should have developed on the aortic right coronary cusp remains unclear though it may have related to the underlying amyloid infiltration of the right coronary leaflet. Endothelial damage predisposes to platelet adhesion, aggregation, and thrombus formation through a reduction in local nitric oxide production ${ }^{67}$ and the undoubted loss of normal valve architecture that resulted from amyloid infiltration may have led to dysfunction of the overlying endothelium. In view of the close anatomical relation between the aortic valve thrombus and right coronary ostium.

We assumed that a coronary embolus originating from the thrombus on the aortic valve caused the small non- $Q$ wave myocardial infarction. The inferior distribution of the minor electrocardiographic changes was consistent with this. Despite the history of cigarette smoking no surgical or subsequent clinical evidence of underlying obstructive coronary artery disease was found. Preoperative coronary angiography was not performed because of the high risk of systemic embolisation from the aortic valve thrombus during right coronary engagement. In patients aged less than 35 acute myocardial infarction secondary to atheromatous coronary artery disease is rare whereas coronary embolism and/or unusual underlying structural abnormalities are more common. ${ }^{89}$ Young patients presenting with acute ischaemic syndromes need to be carefully assessed and all of them should be examined by echocardiography.

Histological and immunocytochemical examination indicated the presence of primary or AL type amyloid deposits within the aortic right coronary cusp. ${ }^{4}$ Amyloid infiltration of heart valves has been described in association with generalised primary amyloidosis. ${ }^{10}$ In our patient there was no clinical evidence or family history of amyloidosis. Rare cases of isolated valvar amyloid have been reported in which the affected valves had a nodular and warty appearance, ${ }^{3}$ but these were discovered incidentally at necropsy and were not associated with thrombosis or clinical sequelae. Microscopic amyloid deposits are a common and non-specific finding in chronically scarred and calcified valves (so-called dystrophic amyloid). ${ }^{211}$ It is unusual to find isolated primary amyloid in a bicuspid aortic valve that affects the aortic right coronary cusp alone and is not associated with valvar calcification or scarring.

In summary, amyloid infiltration was found in a non-calcified, congenitally bicuspid aortic valve where it was restricted to the 
right coronary cusp and was associated with overlying organised thrombus. Aortic valve obstruction resulted and coronary embolism led to acute myocardial infarction. We believe this to be the first reported case of primary valvar amyloid presenting with clinical sequelae. The case also emphasises the need for careful assessment and in particular echocardiography in all young patients presenting with acute ischaemic syndromes.

1 Falk E, Ladefuged C, Christensen HE. Amyloid deposits in calcified aortic valves. Acta Path Microbiol Scand Sect A 1981;89:23-6.

2 Cooper JH. Localized dystrophic amyloidosis of heart valves. Virchows Arch 1983;14:649-53.

3 Pomerance A. Acquired non-rheumatic valvular and endocardial pathology. Pathol Annu 1977 (Part 2); 151-87.

4 Romhanyi G. Differences in ultrastructural organization of amyloid as revealed by sensitivity or resistance to induced proteolysis. Virchows Arch 1972;357:29-52.
5 Landrum AC, Slidders W, Fraser DS. Renal hyalin: a study of amyloidosis and diabetic fibrinous vasculosis with new staining methods. $\mathcal{F}$ Clin Pathol 1972;25: with new

6 Badimon L, Badimon J,. Gaivez A, Chesebro JH, Fuster $\mathrm{V}$. Influence of arterial damage and wall shear rate on platelet deposition: Ex viro study in a swine model. Arterioscl Thromb 1986;6:3.12-20.

7 Groves PH, Penny WJ, Cheadle HA, Lewis MJ. Exogenous nitric oxide inhibits in vivo platelet adhesion following balloon angioplasty. Cardiovasc Res 1992;26: 615-9.

8 Betriu A, Castaner A, Sanz GA, Carlos Pare J, Roig E, Coll S, et al. Angiographic findings 1 month after myocardial infarction: A prospective study of 259 survivors. Circulation 1982;65:1099-105.

9 Glover MU, Kuber MT, Warren SE, Vieweg WVR. Myocardial infarction before age 36: Risk factor and Myocardial infarction before age 36 : Risk factor and arteriograph

10 Koletsky S, Stecher RM. Primary systemic amyloidosis: involvement of cardiac valves, joints and bones with involvement of cardiac valves, joints and bones with
pathologic fracture of the femur. Arch Pathol Lab Med pathologic fracture

11 Goffin YA. Microscopic amyloid deposits in the heart valves: A common local complication of chronic damage and scarring. $₹$ Clin Pathol 1980;33:262-8. 